\title{
Oral health status of psoriatic patients managed with modern biological therapy
}

\author{
Maria Olejnik ${ }^{1}$, Kinga Adamska², Zygmunt Adamski², Barbara Dorocka-Bobkowska ${ }^{1}$ \\ 1Department of Gerodontology and Oral Pathology, Poznan University of Medical Sciences, Poznan, Poland \\ ${ }^{2}$ Department and Clinic of Dermatology, Poznan University of Medical Sciences, Poznan, Poland
}

Adv Dermatol Allergol 2022; XXXIX (6): 1151-1156

DOI: https://doi.org/10.5114/ada.2021.112317

\begin{abstract}
Introduction: Psoriasis is a chronic multifactorial dermatosis with an autoimmune and genetic background, with characteristic periods of remission and exacerbation, affecting up to $3 \%$ of the population; it is currently regarded as a systemic disease.

Aim: To investigate the oral health status, including oral mucosal lesion frequency and prosthetic treatment requirements, in psoriatic patients managed with biological therapies.

Material and methods: Forty-two patients diagnosed with psoriasis and managed with biologics were enrolled in this study. All subjects completed a general medical history and a Dermatology Life Quality Index (DLQI) questionnaire. The disease severity was assessed using the Psoriasis Area and Severity Index (PASI) scale and a DLQI questionnaire. To evaluate the oral health status, the following techniques were used: Approximal Plaque Index (API), the Community Periodontal Index (CPI), and the Decayed, Missing, and Filled Teeth (DMFT) index. The oral mucosa evaluation was based on the clinical appearance, location, and morphology of the lesions.

Results: The most common oral lesions in patients with psoriasis were found to be fissured tongue and white coated tongue. Thirteen patients (31\%) presented with clinically healthy oral mucosa. Type II (diffuse type) denture stomatitis (DS) was most common amongst psoriatic patients wearing acrylic removable dentures. Most of the subjects required prosthetic treatment (57.1\%) and the removal of calculus (40.5\%); however, no one required surgical treatment. Conclusions: Fissured tongue seemed to be associated with skin lesions in psoriatic patients managed with biological therapies. The need for dental treatment was observed in $71.4 \%$ of the patients, with most subjects requiring prosthetic treatment. The study indicated that the diffuse type of DS occurred amongst subjects with psoriasis, who were wearers of acrylic removable dentures.
\end{abstract}

Key words: oral mucosa lesions, psoriasis, biological therapy, quality of life.

\section{Introduction}

Psoriasis is a chronic multifactorial dermatosis with an autoimmune and genetic background, with characteristic periods of remission and exacerbation, affecting up to $3 \%$ of the population; it is currently regarded as a systemic disease [1-3]. The aetiology of the disease remains unclear; however, excessive activation of T-helper lymphocytes, which differentiate depending on the stimulus and cause excessive proliferation and differentiation of epidermal keratinocytes, is regarded as an essential factor in disease development [3, 4]. Various local and systemic factors such as trauma, stress, obesity, streptococcal infections, and tobacco smoking may trigger the onset or the exacerbation of the disease $[1,2,5]$. Five types of psoriasis have been described: plaque, guttate, pustular, inverse, and erythrodermic [5].

Management of the disease includes topical therapy with corticosteroids, vitamin $\mathrm{D}_{3}$ derivatives, or calcineurin inhibitors in the case of mild psoriasis, and phototherapy or systemic therapy (cyclosporine, fumarates, methotrexate, and retinoids) in moderate-to-severe cases. A better understanding the immunological background of the disease and developments in biotechnology have enabled the introduction of new drugs, such as proteins that target a selected stage of the immune response; in addition, anti-TNF- $\alpha$ inhibitors, interleukin blockers, and T-cell agents are being used [3-8].

Although the oral cavity is considered a potential source of inflammation that influences overall health

Address for correspondence: Barbara Dorocka-Bobkowska, MD, DDS, PhD, Department of Gerodontology and Oral Pathology, Poznan University of Medical Sciences, 70 Bukowska St, 60-812 Poznań, Poland, phone: +48 6185472 02, e-mail: bdorocka@ump.edu.pl Received: 8.12.2021, accepted: 19.12.2021. 
and may impact skin conditions, minimal data describe the oral health condition and dental needs in psoriatic patients.

\section{Aim}

The aim of this study was to investigate the oral health status including oral mucosal lesion frequency and dental treatment requirements in psoriatic patients managed with biological therapies.

\section{Material and methods}

Forty-two patients diagnosed with psoriasis and undergoing biological treatment in the Dermatology Department of Poznan University of Medical Sciences were enrolled in this study. The data collection took place from November 2018 to September 2019. The inclusion criterion involved a therapeutic regimen for at least 12 weeks before examination in order to evaluate the effects of the treatment. Every subject who qualified for biological therapy was obliged to present a dental certificate, indicating the absence of a chronic inflammatory process in the oral cavity in order to qualify for therapy.

Patients with no definitive diagnosis of psoriasis and who did not sign the informed consent form were excluded.

A qualified dental specialist experienced in oral disease diagnostics and treatment performed a detailed oral examination consisting of anamnesis and clinical evaluation of all the recruited subjects. The history of the patients included age, gender, dental hygiene habits, subjective complaints related to the oral cavity, and addictions. Oral cavity examinations were performed in artificial light with a dental mirror and a WHO periodontal probe.

\section{A}

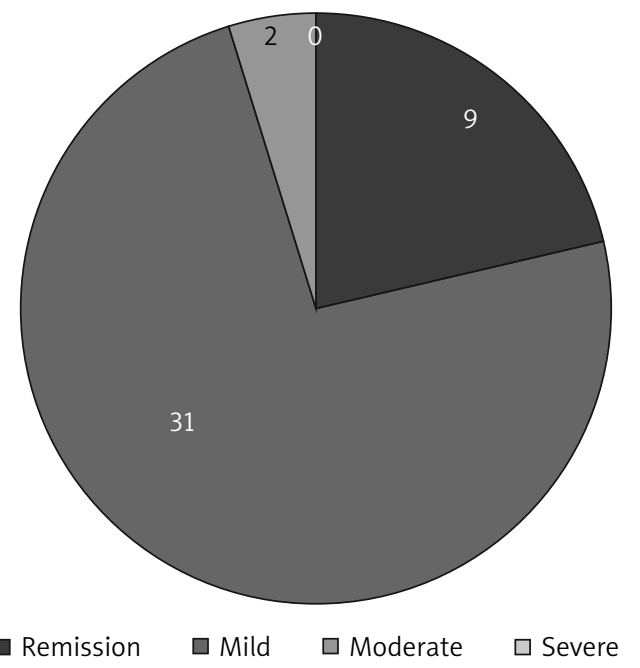

The following indices were used to evaluate the oral hygiene, and the dental and the periodontal status: Approximal Plaque Index according to Lange et al. (API), the Decayed, Missing, and Filled Teeth (DMFT) index, and the Community Periodontal Index (CPI) $[9,10]$. The oral mucosa evaluation was based on the appearance of the lesions, their location, and morphology. Denture stomatitis (DS) was categorized according to the Newton classification and described as Type 1, Type 2, or Type 3.

The Psoriasis Area and Severity Index (PASI) score was used to assess the severity of the disease as remission (score 0), mild (<10), moderate (10-18), or severe (>18). Additionally, all patients completed a general medical history and Dermatology Life Quality Index (DLQI) questionnaire, which classified the effect of the disease on the quality of life as none (score $0-1$ ), small (2-5), moderate (6-10), very large (11-20), and extremely large (21-30) [11].

\section{Results}

The study group comprised 24 men and 18 women, with an average age of 47 years (range: 20-69 years, $\mathrm{SD}=24.5)$. The mean duration of the disease was 28 years (range: $5-60$ years, SD $=27.5$ ). The majority of the group suffered from psoriasis vulgaris (27; 64.3\%), whereas co-occurrence of psoriatic arthritis appeared in 15 cases (35.7\%). Half of the participants reported a positive family history of psoriasis. Most patients (30; 71.4\%) received tumour necrosis factor (TNF) inhibitors. The biological management was as follows: adalimumab (20), ustekinumab (7), infliximab (6), secukinumab (5), golimumab (2), and etanercept (2).

According to the severity of psoriasis, most patients were classified as mild, and in most cases, the disease had no or little effect on their quality of life (Figure 1).
B

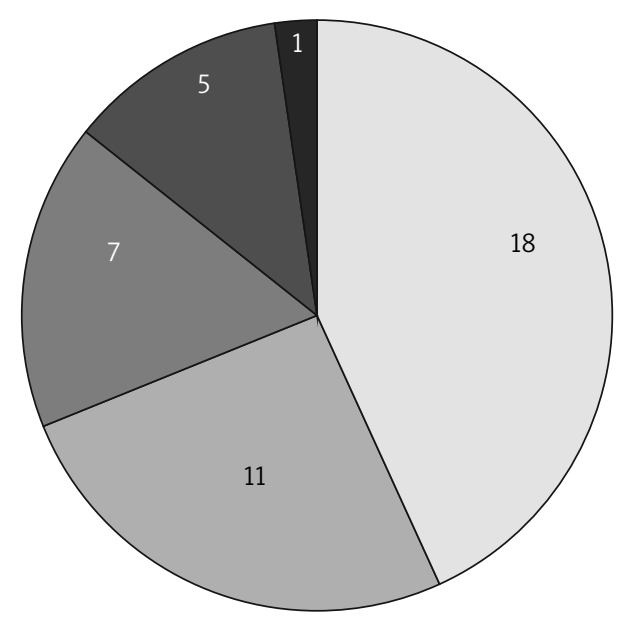

$\square$ No effect $\square$ Small effect $\quad \square$ Large effect $\square$ Very large effect $\quad$ Extremely large effect

Figure 1. The distribution of study population according to the severity of psoriasis (PASI scale, A) and the effect on the quality of life (DLQI, B) 
Twelve subjects (28.6\%) smoked tobacco. The mean duration of tobacco use was 17 years (range: $5-40$ years, SD $=10.1)$. Nine patients $(21.4 \%)$ used acrylic removable denture appliances, and 1 of them also wore the denture during the night. The types of appliances used are presented in Table 1.

Most of the patients used acrylic denture appliances; only 3 of them used frameworks.

Three $(7.1 \%)$ subjects were diagnosed with denture-related stomatitis (DS), and 1 patient (2.4\%) presented with angular cheilitis. In the present study we found that the diffuse type of inflammation was most frequently observed in psoriatic patients, the lesion being located on the hard palate and confined to the mucosa in contact with the acrylic denture plate.

Burning sensation of the mouth and dryness of the oral mucosa were found to be frequent complaints in DS.

Only 2 patients (4.8\%) from the study group reported subjective complaints, which included: gum bleeding (1 patient; $2.4 \%$ ) and a burning sensation of the tongue $(1 ; 2.4 \%)$

The oral health status was determined from the following indices: Approximal Plaque Index according to Lange et al. (API), the Decayed, Missing, and Filled Teeth (DMFT) index, and the Community Periodontal Index (CPI). The results are shown in Table 2.

The mean value of the Approximal Plaque Index was 69.5, which indicates average hygiene in the study group, which requires improvement. The mean value of decayed teeth (D) was 0 , pointing to good caries management and resulting in a low need for conservative treatment. On the other hand, the high mean value of missing teeth $(M)$ required many prosthetic interventions.

Oral mucosal lesions were present in 29 individuals (69\%). The most commonly found alterations included the following: fissured tongue (18 cases, 42.9\%), white coated tongue (11 cases; $26.2 \%$ ), and linea alba (9 cases; $21.4 \%$ ). Thirteen patients (31\%) presented with clinically healthy oral mucosa. In some cases, more than one type of pathologic change was observed in a subject concurrently.

Table 3 shows the prevalence of oral mucosal lesions in the study population. Fissured tongue was the most commonly observed oral pathologic lesion.

Table 2. The oral health status of psoriatic patients managed with biologic therapy

\begin{tabular}{lcccc}
\hline Parameter & Mean & Min. & Max. & SD \\
\hline D & 0 & 0 & 4 & 1.0 \\
\hline M & 13 & 0 & 32 & 8.5 \\
\hline F & 7 & 0 & 15 & 4.2 \\
\hline DMFT & 20 & 4 & 32 & 6.5 \\
\hline API & 69.5 & 18 & 100 & 21.5 \\
\hline CPI & 2.5 & 0 & 4 & 1.1
\end{tabular}

Table 1. The types of removable denture appliances used by the psoriatic patients

\begin{tabular}{ll}
\hline Removable dentures - acrylic and frameworks & 9 \\
\hline Complete denture in the maxilla & 3 \\
\hline $\begin{array}{l}\text { Complete denture in the maxilla and acrylic } \\
\text { partial denture in the mandible }\end{array}$ & 2 \\
\hline Complete denture in the maxilla and mandible & 1 \\
\hline Framework in the maxilla and mandible & 3 \\
\hline
\end{tabular}

Fissured tongue was a common manifestation of the tongue observed in patients with psoriasis. It is distinguished by a deep, prominent groove on the dorsum of the tongue (Figure 2).

Type II (diffuse type) of DS was found most frequently amongst patients with psoriasis who were also wearers of acrylic removable dentures. All patients exhibited a lesion in the upper jaw - type 2 of DS in subjects with psoriasis, as illustrated in Figure 3.

The need for dental treatment was observed in 30 (71.4\%) patients. In some cases, more than one dental procedure was recommended. Most of the subjects required prosthetic treatment (24 cases; $57.1 \%$ ) and the removal of calculus (17 patients; $40.5 \%$ ). Also, a prevalence for caries management was noticed (12 patients; $28.6 \%)$; however, no one required surgical treatment (Table 4).

\section{Discussion}

A better understanding of the immunological background of autoimmune inflammatory diseases and the development of biotechnology has enabled the intro-

Table 3. The prevalence of mucosal lesions in patients with psoriasis managed with biologic therapy $(N=42)$

\begin{tabular}{lc}
\hline Parameter & $n(\%)$ \\
\hline Fissured tongue & $18(42.9)$ \\
\hline White coated tongue & $11(26.2)$ \\
\hline Linea alba & $9(21.4)$ \\
\hline Geographic tongue & $2(4.7)$ \\
\hline Denture stomatitis I & 0 \\
\hline Denture stomatitis II & $2(4.8)$ \\
\hline Denture stomatitis III & $1(2.4)$ \\
\hline Nicotine stomatitis & $1(2.4)$ \\
\hline Melanotic macule & $1(2.4)$ \\
\hline Angular cheilitis & $1(2.4)$ \\
\hline No lesions observed & $13(31.0)$ \\
\hline
\end{tabular}




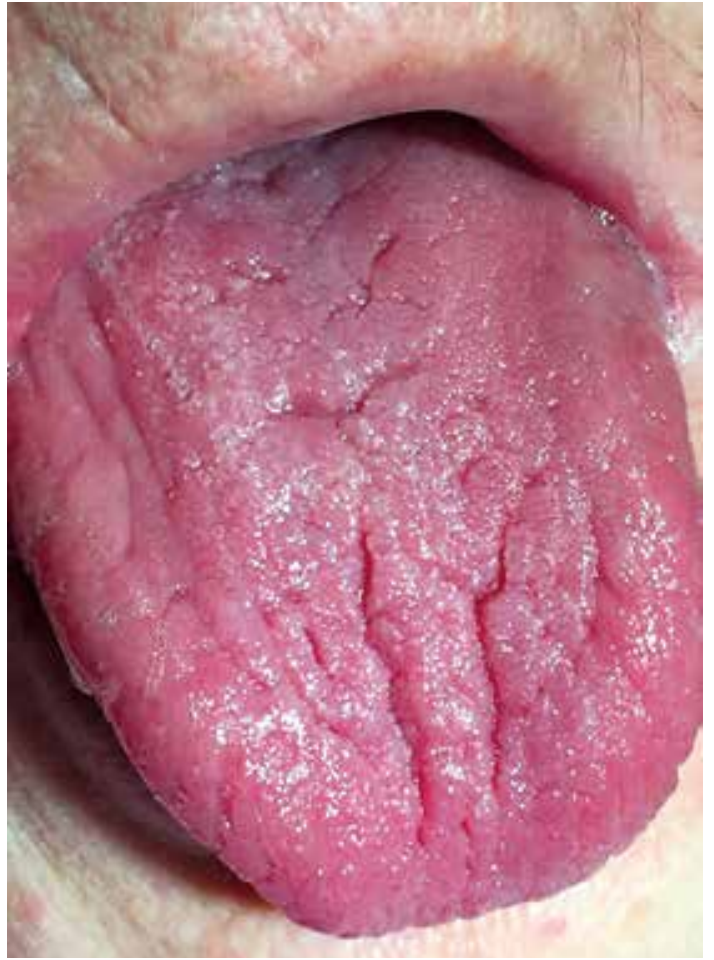

Figure 2. Fissured tongue in patient with psoriasis

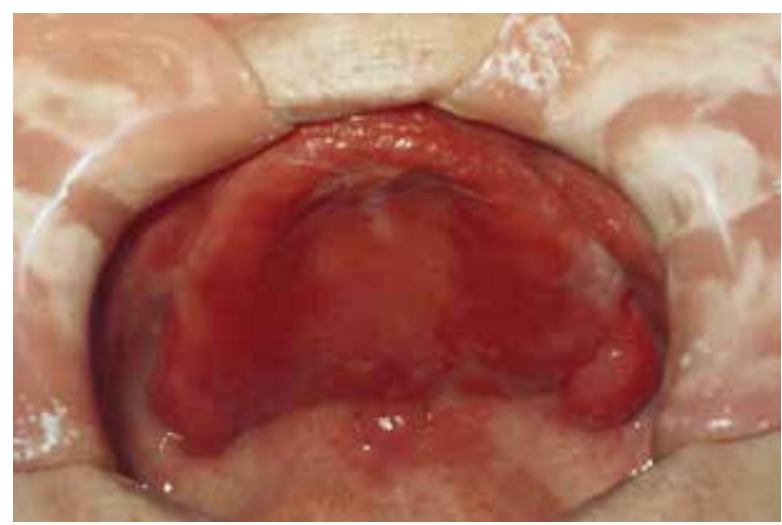

Figure 3. Type 2 DS, diffuse hyperaemic mucosa extending over the entire denture-bearing area in an acrylic complete denture wearer with psoriasis

Table 4. Dental treatment needs of psoriatic patients managed with biologic therapy $(N=42)$

\begin{tabular}{lc}
\hline Parameter & $n(\%)$ \\
\hline Dental needs & $30(71.4)$ \\
\hline Scaling & $17(40.5)$ \\
\hline Conservative treatment & $12(28.6)$ \\
\hline Surgical treatment & 0 \\
\hline Prosthetic treatment & $24(57.1)$ \\
\hline Periodontal treatment & $2(4.8)$ \\
\hline
\end{tabular}

duction of new drugs - such as proteins with a target at selected stage of the immune response. Excessive activation of T-helper lymphocytes, which differentiate depending on the stimulus and cause excessive proliferation and differentiation of epidermal keratinocytes, is essential in psoriasis development. IL-12 is responsible for the differentiation of Th1 cells, whereas IL-23 influences the maturation of Th17 lymphocytes, which are also associated with inflammatory bowel diseases, rheumatoid arthritis, multiple sclerosis, and inflammatory response in obesity. Large molecular-weight proteins, administered as injections in targeted therapy, are known as biologics and include receptor fusion proteins and monoclonal antibodies [3, 4, 8]. They were introduced in psoriasis treatment in 2002 and led to significant improvement in management efficacy [12].

With its high microbiome content, the oral cavity is considered a potential source of inflammation that influences overall health and may impact skin conditions and provoke the first manifestation or exacerbation of psoriasis. The structural similarities between streptococcal $M$ protein and epidermal keratins inducing cross-reactivity are present in the psoriasis pathogenesis $[13,14]$.

The association between psoriasis and periodontitis is described in the literature and even includes periodontitis as a new comorbidity of psoriasis. The elevated levels of cytokines, such as tumour necrosis factor- $\alpha$ (TNF- $\alpha$ ), IL-17, and IL-22, are described in the pathogenesis of the periodontal disease, which are also targeted by biologics. That may lead to the improvement of periodontal parameters in patients managed with these new drugs [15-18].

There are minimal data on the oral health condition in psoriatic patients. However, some authors have reported a high number of missing teeth in this population [18-20]. Our study seems to confirm this observation, as more than half of subjects required prosthodontic treatment, probably as a result of the removal of the inflammation foci before the commencement of therapy, which is essential in order to avoid harmful side effects of biological agents and the exacerbation of psoriasis $[3,13]$.

The use of removable denture appliances, especially for a prolonged period, may lead to the overgrowth of Candida albicans, a fungus that is one of the many oral microflorae [21-23]. In our study, type II of DS - diffuse hyperaemic mucosa extending over the entire denturebearing area - was found most frequently amongst patients with psoriasis. All patients exhibited a lesion in the upper jaw. This may be attributed to the isolation of oral mucosa tissues under a maxillary complete denture, particularly when the maxillary denture forms a good seal, resulting in its own microenvironment, disturbing the normal microbiological balance in the space. Additionally, DS in the maxilla may be associated with the amount of tissue covered by the maxillary denture plate and its strong retention [23]. 
It is advantageous to administer a combination of miconazole and antimicrobial photodynamic therapy to manage Candida biofilm in patients with Candida-associated DS [24, 25].

This condition may spread the infection from the denture-covered oral mucosa to the angles of the mouth. It is assumed that in angular cheilitis, the lesions are infected by either Candida albicans and Staphylococcus aureus, or both [23-25].

The oral mucosal lesions associated with psoriasis are mainly limited to the tongue. Fissured tongue described as the presence of anteroposteriorly or laterally oriented fissures on the dorsal surface of the tongue affects $2-5 \%$ of the general population and seems to be the most common oral lesion in psoriatic patients [26-29]. The geographic tongue, characterized as well-demarcated zones of erythema at dorsal tongue mucosa, because it is associated with increased levels of TNF- $\alpha$ and IL-6, occurs less frequently in a population managed with biologicals $[26,30]$.

This study presents the oral health status in psoriatic patients managed with modern biological drugs. The elimination of foci inflammation led to loss of teeth, which resulted in an urgent need for prosthetic intervention. Further investigation, including the measurement of periodontal parameters and serum and saliva levels of inflammation markers, is required to confirm the influence of the drugs on the periodontal condition. A detailed oral mucosa examination performed before and after the biologic treatment may be necessary to verify the improvement of the tongue condition. Because the oral cavity is a potential source of inflammation, which may enhance psoriasis, meticulous oral and denture hygiene in addition to regular dental visits should be recommended to all psoriatic patients.

\section{Conclusions}

This study showed that fissured tongue seemed to be associated with skin lesions in psoriatic patients managed with biological therapies. The study also indicated that the diffuse type of denture stomatitis occurred amongst subjects with psoriasis, who were also wearers of acrylic removable dentures. Moreover, the need for dental treatment was observed in $71.4 \%$ of the patients, with most subjects requiring prosthetic treatment. The use of dental indexes to assess oral health should be recommended in addition to standardized Psoriasis Area and Severity Index (PASI) and general medical history of psoriatic patients.

\section{Conflict of interest}

The authors declare no conflict of interest.

\section{References}

1. Christophers E. Psoriasis - epidemiology and clinical spectrum. Clin Exp Dermatol 2001; 26: 314-20.

2. Griffiths CE, Barker JN. Pathogenesis and clinical features of psoriasis. Lancet 2007; 370: 263-71.

3. Reich A, Adamski Z, Chodorowska G, et al. Psoriasis. Diagnostic and therapeutic recommendations of the Polish Dermatological Society. Part 2. Dermatol Rev 2020; 107: 110-37.

4. Owczarczyk-Saczonek A, Placek W. Psoriasis as an autoimmune disease. Dermatol Rev 2014; 101: 278-87.

5. Boehncke WH, Schön MP. Psoriasis. Lancet 2015; 386: 983-94.

6. Menter A, Griffiths CE. Current and future management of psoriasis. Lancet 2007; 70: 272-84.

7. Osmola-Mańkowska A, Teresiak-Mikołajczak E, SkrzypczakZielińska M, Adamski Z. Genetic polymorphism in psoriasis and its meaning for the treatment efficacy in the future. Adv Dermatol Allergol 2018; 35: 331-7.

8. Rønholt K, Iversen L. Old and new biological therapies for psoriasis. Int J Mol Sci 2017; 18: 2297.

9. Dhingara K, Vandana KL. Indices for measuring periodontitis: a literature review. Int Dent J 2011; 61: 76-84.

10. Broadbent JM, Thomson WM. For debate: problems with the DMF index pertinent to dental caries data analysis. Community Dent Oral Epidemiol 2005; 33: 400-9.

11. Oji V, Luger TA. The skin in psoriasis: assessment and challenges. Clin Exp Rheumatol 2015; 33: S14-9.

12. Reid C, Griffiths CEM. Psoriasis and treatment: past, present and future aspects. Acta Derm Venereol 2020; 100: adv00032.

13. Macklis P, Adams K, Kaffenberger J, et al. The association between oral health and skin disease. J Clin Aesthet Dermatol 2020; 13: 48-53.

14. Vieira C, Caramelli B. The history of dentistry and medicine relationship: could the mouth finally return to the body? Oral Dis 2009; 15: 538-46.

15. Mendes VS, Cota LOM, Costa AA, et al. Periodontitis as another comorbidity associated with psoriasis: a case-control study. J Periodontol 2019; 90: 358-66.

16. Peddis N, Musu D, Ideo F, et al. Interaction of biologic therapy with apical periodontitis and periodontitis: a systematic review. Aust Dent J 2019; 64: 122-34.

17. Ding C, Ji X, Chen X, et al. TNF- $\alpha$ gene promoter polymorphisms contribute to periodontitis susceptibility: evidence from 46 studies. J Clin Periodontol 2014; 41: 748-59.

18. Zenobia C, Hajishengallis G. 2015. Basic biology and role of interleukin-17 in immunity and inflammation. Periodontology 2000; 69: 142-59.

19. Fadel HT, Flytström I, Calander AM, et al. Profiles of dental caries and periodontal disease in individuals with or without psoriasis. J Periodontol 2013; 84: 477-485.

20. de Barros FC, Sampaio JN, da Figueredo CM, et al. Higher prevalence of periodontitis and decayed, missing and filled teeth in patients with psoriasis. Eur J Dent 2020; 14: 366-70.

21. Singh A, Verma R, Murari A, Agrawal A. Oral candidiasis: an overview. J Oral Maxillofac Pathol 2014; 18: S81-5.

22. Dorocka-Bobkowska B, Düzgüneş N, Konopka K. AmBisome and Amphotericin B inhibit the initial adherence of Candida albicans to human epithelial cell lines but do not cause yeast detachment. Med Sci Monit 2009; 15: 262-9.

23. Dorocka-Bobkowska B, Medynski D, Prylinski M. Recent advances in tissue conditioners for prosthetic treatment. A review. Adv Clin Exp Med 2017; 4: 723-8. 
24. Davies A, Gebremedhin S, Yee M, et al. Cationic porphyrinmediated photodynamic inactivation of Candida biofilms and the effect of miconazole. J Physiol Pharmacol 2016; 67: 777-83.

25. Gebremedhin S, Dorocka-Bobkowska B, Prylinski M, et al. Miconazole activity against Candida biofilms developed on acrylic discs. J Physiol Pharmacol 2014; 65: 593-600.

26. Olejnik M, Osmola-Mańkowska A, Ślebioda Z, et al. Oral mucosal lesions in psoriatic patients based on disease severity and treatment approach. J Oral Pathol Med 2020; 49: 822-8.

27. Costa AA, Cota LOM, Mendes VS, et al. Impact of oral lesions on the quality of life of psoriatic individuals: a case-control study. Oral Dis 2021; 27: 1813-21.

28. Daneshpazhooh M, Moslehi H, Akhyani M, Etesami M. Tongue lesions in psoriasis: a controlled study. BMC Dermatol 2004; 4: 16

29. Hernández-Pérez F, Jaimes-Aveldańez A, Urquizo-Ruvalcaba ML, et al. Prevalence of oral lesions in patients with psoriasis. Med Oral Patol Oral Cir Bucal 2008; 13: E703-8.

30. Alikhani M, Khalighinejad N, Ghalaiani P, et al. Immunologic and psychologic parameters associated with geographic tongue. Oral Surg Oral Med Oral Pathol Oral Radiol 2014; 118: 68-71. 